\title{
Semi-inclusive $\Lambda$ production and generalized sullivan processes
}

\author{
W-Y. P. Hwang ${ }^{1,2}$, Chih-Yi Wen ${ }^{1,2}$ \\ ${ }^{1}$ Department of Physics, National Taiwan University, Taipei, Taiwan 106, R.O.C. \\ 2 Center for Theoretical Physics, Laboratory for Nuclear Science and Department of Physics, Massachusetts Institute of Technology, Cambridge, MA 02139, \\ USA.
}

Received: 7 March 1996 / Revised version: 30 December 1996

Communicated by W. Weise

\begin{abstract}
In this paper, we suggest that semi-inclusive $\Lambda$ production with a high energy electron or muon beam may be employed as an effective way to pin down the possible importance of the generalized Sullivan process, in which the virtual photon may strike and smash the meson in the cloud surrounding the target proton. The role of such generalized Sullivan processes in deep inelastic scattering (DIS) has been exploited recently in connection with the observed violation of the Gottfried sum rule as well as with the so-called "proton spin crisis". Our present numerical results indicate that, in the kinematic region characterized typically by small $x$, large $z$, and a small specific range of $y$ (with the parton variables $x, y$, and $z$ defined in the standard manner), the contribution from Sullivan processes may indeed be of numerical importance as compared to that due to the fragmentation process.
\end{abstract}

PACS: $13.75 . J z ; 13.60 . \mathrm{Hb} ; 13.15 . \mathrm{Em}$.

\section{Introduction}

Nowadays there is little doubt that strong interactions are described by quantum chromodynamics [QCD]. QCD is asymptotically free so that the theory has been tested, successfully to some extent, in high energy processes. To use QCD to describe hadron properties at low and intermediate energies, however, it has become of increasing importance (and also of increasing complication) to be able to separate clearly the nonperturbative aspects of QCD from its perturbative parts.

One of the potentially very important nonperturbative aspects has to do with pions and other low-lying pseudoscalar mesons, often recognized as the Goldstone bosons arising from breaking of $S U\left(N_{f}\right)_{L} \times S U\left(N_{f}\right)_{R}$ chiral symmetry in QCD for $N_{f}$ flavors of almost massless quarks into the $S U\left(N_{f}\right)_{V}$ symmetry. These (Goldstone) particles couple to, e.g., nucleons nonperturbatively and it has become clear that such couplings cannot be reproduced in any existing perturbative QCD calculations. Thus, effects caused by the pion clouds associated with nucleons (or with other hadrons) are likely nonperturbative in nature and would have to be carefully identified in any effort of isolating nonperturbative aspects from QCD. One imperative question is whether it is possible to go over to high energy limits so as to avoid dealing directly with such nonperturbative pion exchange effect. As we shall see, the answer to such important question may be highly nontrivial.

An important observation has in fact been made by Sullivan [1] in 1972 that in deep-inelastic-scattering (DIS) of a nucleon by leptons, the process in which the virtual photon strikes the pion emitted by the nucleon and smashes the pion into debris, scales like the original process where the virtual photon strikes and smashes the nucleon itself. In other words, the process will contribute by a finite amount to cross sections in the Bjorken scaling limit, i.e., the standard high energy limit as characterized by $Q^{2} \rightarrow \infty$ and $\nu \equiv p \cdot q / m_{p} \rightarrow \infty$ with $x \equiv Q^{2} /\left(2 m_{p} \nu\right)$ held fixed. Following Sullivan's derivation [1], we have, for the process depicted pictorially by Fig. 1a but with $K^{+}$and $\Lambda$ replaced by $\pi$ and $N$, respectively,

$$
\begin{aligned}
\delta F_{2 N}^{\pi}\left(x, Q^{2}\right)= & \int_{x}^{1} d y f_{\pi}(y) F_{2 \pi}\left(\frac{x}{y}, Q^{2}\right) \\
f_{\pi}(y)= & \frac{3}{16 \pi^{2}}\left(\frac{f_{\pi N N} 2 m_{N}}{\mu}\right)^{2} y \\
& \int_{-\infty}^{t^{m}} d t \frac{(-t)\left|F_{\pi}(t)\right|^{2}}{\left(-t+\mu^{2}\right)^{2}}
\end{aligned}
$$

with $t^{m}=-\frac{m_{N}^{2} y^{2}}{(1-y)}$. Here $m_{N}$ and $\mu$ are, respectively, the nucleon mass and the pion mass. $F_{2 \pi}(x)$ is the pion structure function, as would be measured in DIS with the pion as the target. $\delta F_{2 N}(x)$ is the correction to the nucleon structure function due to the specific Sullivan process. $f_{\pi}(y)$ is the probability of finding a pion with momentum fraction $y$ of the nucleon's momentum. $F_{\pi}(t)$ is the form factor which characterizes the $t$-dependence of the $\pi N N$ coupling $f_{\pi N N}$.

It is of great interest to look into the structure of (1) and (2) since it indicates that the correction $\delta F_{2 N}(x)$ does not vanish in the Bjorken limit as long as the pion structure function $F_{2 \pi}\left(x, Q^{2}\right)$ does not vanish much more rapidly compared to the nucleon structure function $F_{2 N}(x)$ (which would be naturally the case). As we shall see later in the paper, (1) and (2) may also be verified using the light-cone perturbation theory 
so that little doubt could be cast into the validity of (1) and (2). Nevertheless, this result has the severe implication, an implication largely ignored by the high energy physics community so far, that the pionic effect addressed here appears to be one of the nonperturbative QCD effects which cannot be thrown away by simply going over to the high energy limit.

The pion exchange effect indicated by (1) and (2) has been employed by many authors in a variety of contexts. For instance, Thomas and Frankfurt et al. [2] have attempted to attribute the observed asymmetry $\frac{1}{2}[\bar{u}(x)+\bar{d}(x)]-\bar{s}(x)$ to Sullivan processes and obtained a nucleon bag radius of about 0.8 fermi or a monopole $\pi N N$ form factor of about $500 \mathrm{MeV}$ (an unusally soft form factor). On the other hand, the so-called EMC effect, observed originally by the EMC collaboration [3] and later confirmed by others [4-6], may be explained by the pion-excess picture [7-10] which is based upon a treatment of the Sullivan process in nuclear medium (as contrasted with that in free space). Recently, Henley and Miller and several other authors [11-13] have related Sullivan processes to the violation of the Gottfried sum rule [14], as observed by the NMC group [15]. In addition, much interest has been generated in the community by the experiments [16] to pin down the nucleon spin structure functions - leading to some puzzle often referred to as the "proton spin crisis". It was pointed out [17] that such puzzle can also be understood as depolarization of the valent quark spins by the presence of pseudoscalar Goldstone bosons (thus forcing the core to reverse its spin).

Of course, it may be argued that in all the experiments cited above the relevant $Q^{2}$ is typically in the range of a few $\mathrm{GeV}^{2}$ up to a little over $10 \mathrm{GeV}^{2}$ which may not be high energy enough and thus cannot easily deny the meson-exchange interpretations. However, the fact that such meson-exchange interpretations work so well should already be quite disturbing to most high energy theorists, especially those who has ignored completely the nonperturbative pion exchange effects until now - not mentioning that the contribtions from such processes may actualy persist in the very high energy limit. For this sake, it is therefore of importance to examine in the near future if there are other important manifestations of the nonperturbative meson-exchange effect in high energy processes which can be tested experimentally and should be isolated carefully in future theoretical works. To this end, we choose in this paper to consider, as an important example, the semiinclusive $\Lambda$ production in deep inelastic scattering (DIS) by a charged lepton beam $(e$ or $\mu)$ :

$l+p \longrightarrow l^{\prime}+\Lambda+X$,

Here for the sake of definiteness the semi-inclusive $\Lambda$ production with the proton target is considered. At this juncture, we wish to mention that some authors [18] have also investigated the pion contribution to semi-inclusive deep inelastic scattering with slow baryons ( $N$ 's or $\Delta$ 's) in the final states, an investigation related but complimentary to the present work which we shall mention briefly later in the paper.

In contrast with the idea of a parton model in which a hadron consists of partons of different kinds, the fragmentation hypothesis of Field and Feynman [19] states that a high-energy parton of a specific kind (a quark, an antiquark, or a gluon) has different probabilities of fragmenting into different hadrons. In such fragmentation picture, the fragmentation function $D_{q}^{h}(z)$ is introduced for a given parton of flavor $q$ and the hadron

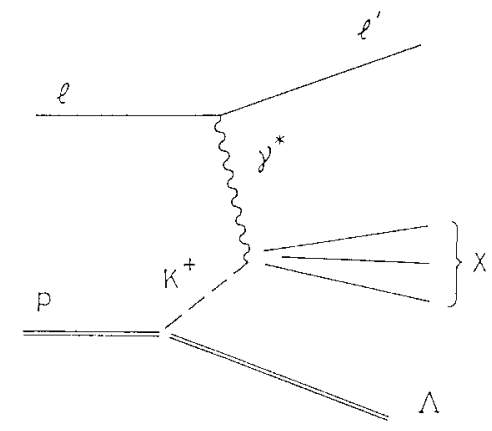

(a)

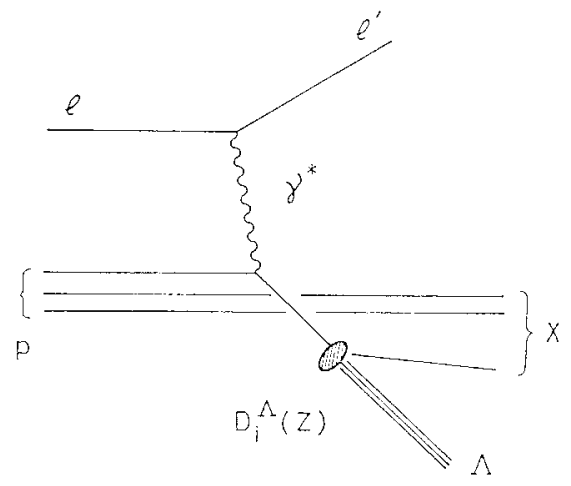

(b)

Fig. 1.a,b. Semi-inclusive production of $\Lambda$ in the deep inelastic scattering of the proton by a charged lepton: a production mechanism through the Sullivan process and $\mathbf{b}$ production mechanism in the fragmentation picture

$h$, with $z$ the fraction of the parton's longitudinal momentum carried away by the hadron. The fragmentation picture has been used in many high energy processes, including semiinclusive hadron production in deep inelastic scattering (DIS), a process as illustrated by Fig. $1 \mathrm{~b}$.

It is clear that the Sullivan process as illustrated by Fig. 1a also provides a mechanism for semi-inclusive production of $\Lambda$ in DIS. In light of the potentially important role played by the Sullivan process in connection with the parton model, it is a natural question to investigate whether the generalized Sullivan process as shown by Fig. 1a may give rise to some important contribution as compared to that given in the fragmentation picture (Fig. 1b). This will be the primary focus of the present paper.

\section{Formulation}

In this section, we wish to derive the basic formulae for describing the Sullivan process as illustrated by Fig. 1a, first using the procedure adopted by Sullivan [1] and then obtaining the same result making use of the alternative method developed by Drell, Levy, and Yan [20]. Following Sullivan [1], we introduce the following variables,

$$
\begin{aligned}
\ell(\ell)+p(p) & \rightarrow \ell^{\prime}\left(\ell^{\prime}\right)+\Lambda\left(p^{\prime}\right)+X\left(p_{n}\right) \\
q & \equiv \ell-\ell^{\prime}, \quad \nu \equiv p \cdot q / m
\end{aligned}
$$




$$
\begin{aligned}
\omega & =\frac{2 p \cdot q}{Q^{2}}=\frac{2 m \nu}{Q^{2}}, \\
s & =(p+q)^{2} \approx Q^{2}(\omega-1) ; \\
k & \equiv p-p^{\prime}, \\
\omega^{\prime} & =\frac{2 k \cdot q}{Q^{2}}=\frac{2 \mu \nu^{\prime}}{Q^{2}}, \\
s^{\prime} & =(k+q)^{2} \approx Q^{2}\left(\omega^{\prime}-1\right), \\
t & \equiv k^{2}=m^{2}+m_{\Lambda}^{2}-2 m E^{\prime},
\end{aligned}
$$

with $\ell, \ell^{\prime}, p, p^{\prime}$, and $p_{n}$ the four-momentum variables and $m$, $m_{\Lambda}$, and $\mu$ the mass of the proton, $\Lambda$, and the kaon, respectively. $E^{\prime}$ is the energy of $\Lambda$.

The amplitude which corresponds to Fig. 1(a) may be written as follows,

$A_{\mu}=g_{K N \Lambda} \frac{\bar{u}\left(p^{\prime}\right) i \gamma_{5} u(p)}{t-\mu^{2}}<X\left(p_{n}\right)\left|J_{\mu}^{e m}(0)\right| K(k)>$,

which is to be used in the calculation of the hadronic tensor.

$$
\begin{aligned}
& W_{\mu \nu}(p, q)=\frac{1}{2 M} \sum_{\text {spin av }} \sum_{X} \int \frac{d^{3} p^{\prime}}{(2 \pi)^{3} 2 p_{0}^{\prime}} \times \\
& \quad \times \prod_{i=1}^{n}\left[\frac{d^{3} p_{i}}{(2 \pi)^{3} 2 p_{i 0}}\right] A_{\mu}^{*} A_{\nu}(2 \pi)^{3} \delta^{4}\left(p_{n}+p^{\prime}-p-q\right) .
\end{aligned}
$$

The spin average part may be calculated easily,

$$
\begin{aligned}
& \sum_{\text {spin av }} A_{\mu}^{*} A_{\nu} \\
& =\frac{1}{2} g_{K N \Lambda}^{2} \frac{-T r\left(\hat{p}^{\prime}+m_{\Lambda}\right) \gamma_{5}(\hat{p}+m) \gamma_{5}}{\left(t-\mu^{2}\right)^{2}} \\
& \quad<K\left|J_{\mu}^{e m}(0)\right| X><X\left|J_{\nu}^{e m}(0)\right| K> \\
& =g_{K N \Lambda}^{2} \frac{\left(m_{\Lambda}-m\right)^{2}-t}{\left(t-\mu^{2}\right)^{2}} \\
& \quad<K\left|J_{\mu}^{e m}(0)\right| X><X\left|J_{\nu}^{e m}(0)\right| K>.
\end{aligned}
$$

The DIS with the kaon as the target would be described by the hadronic tensor,

$$
\begin{aligned}
W_{\mu \nu}^{K}(k, q)= & \frac{1}{2 \mu} \sum_{X} \int \prod_{i=1}^{n}\left[\frac{d^{3} p_{i}}{(2 \pi)^{3} 2 p_{i 0}}\right] . \\
& \cdot<K\left|J_{\mu}^{e m}(0)\right| X><X\left|J_{\nu}^{e m}(0)\right| K> \\
& \cdot(2 \pi)^{3} \delta^{4}\left(p_{n}-k-q\right),
\end{aligned}
$$

so that we may rewrite the hadronic tensor as

$$
\begin{aligned}
W_{\mu \nu}(p, q)= & \frac{\mu}{M} \int \frac{d^{3} p^{\prime}}{(2 \pi)^{3} 2 p_{0}^{\prime}} g_{K N \Lambda}^{2} . \\
& \cdot \frac{\left(m_{\Lambda}-m\right)^{2}-t}{\left(t-\mu^{2}\right)^{2}} W_{\mu \nu}^{K}(k, q) .
\end{aligned}
$$

Using the relation

$d^{3} p^{\prime}=\frac{\pi E^{\prime}}{\omega} d t d \omega^{\prime}$,

we obtain

$$
\begin{aligned}
W_{\mu \nu}(p, q)= & \frac{\mu}{m} \int d t d \omega^{\prime} g_{K N \Lambda}^{2} \frac{\pi}{2 \omega} . \\
& \cdot \frac{\left(m_{\Lambda}-m\right)^{2}-t}{\left(t-\mu^{2}\right)^{2}} W_{\mu \nu}^{K}(k, q) .
\end{aligned}
$$

Using the relation between the hadronic tensor $W_{\mu \nu}$ and the structure function $F_{2}$,

$W_{\mu}^{K \mu}(k, q)=-\frac{\omega^{\prime}}{\mu} F_{2}^{K}\left(\omega^{\prime}\right), \quad W_{\mu}^{\mu}(p, q)=-\frac{\omega}{m} F_{2}(\omega)$.

we obtain

$$
\begin{aligned}
\delta F_{2}(\omega)= & \frac{1}{16 \pi^{2}} \int \frac{d \omega^{\prime} \omega^{\prime}}{\omega^{2}} . \\
& \cdot \int d t \frac{\left(m_{\Lambda}-m\right)^{2}-t}{\left(t-\mu^{2}\right)^{2}} F_{2}^{K}\left(\omega^{\prime}\right) g_{K N \Lambda}^{2}(t) .
\end{aligned}
$$

With suitable substitutions, (11) is just what we have cited as (1) and (2) at the beginning of this paper.

In order to compare this result with what may be obtained from the fragmentation picture, we change the variables $t$ to $z$ (defined as the momentum fraction carried away by the hadron from the parental quark) and express $\omega$ as $x$. We have

$x=\frac{1}{\omega}, \quad z^{\prime}=\frac{\omega^{\prime}}{\omega} \quad$ (kaon's momentun fraction);

$y=\frac{\nu}{E}, \quad z=\frac{E^{\prime}}{\nu}$

$t=m^{2}+m_{\Lambda}^{2}-2 m y z E ;$

so that

$$
\begin{aligned}
d t d \omega^{\prime} & =\left|\frac{\partial\left(t, \omega^{\prime}\right)}{\partial\left(z^{\prime}, z\right)}\right| d z d z^{\prime}=\left|\begin{array}{cc}
-2 m y E & 0 \\
0 & \frac{1}{x}
\end{array}\right| d z d z^{\prime} \\
& =\frac{2 m y E}{x} d z d z^{\prime} .
\end{aligned}
$$

We thus obtain from (11)

$$
\begin{aligned}
\delta F_{2}(x)= & \frac{m^{2} y E}{4 \pi^{2}} \int d z^{\prime} d z \frac{z^{\prime}\left(y z E-m_{\Lambda}\right)}{\left(t-\mu^{2}\right)^{2}} . \\
& \cdot F_{2}^{K}\left(\frac{x}{z^{\prime}}\right) g_{K N \Lambda}^{2}(t) .
\end{aligned}
$$

Using the standard relation [6],

$$
\begin{aligned}
\frac{d^{2} \sigma}{d x d y} & =\frac{\pi \alpha^{2}}{m E x^{2} y^{2}}\left[1+(1-y)^{2}-\frac{m}{E} x y\right] F_{2}(x) \\
& \approx \frac{2 \pi \alpha^{2}}{Q^{2} x y}\left[1+(1-y)^{2}\right] F_{2}(x),
\end{aligned}
$$

we obtain the differential cross section due to the Sullivan process of Fig. 1a,

$$
\begin{aligned}
\frac{d \sigma}{d x d y d z}= & \frac{\pi \alpha^{2}}{m E x^{2} y^{2}}\left[1+(1-y)^{2}\right] \frac{m^{2} y E}{4 \pi^{2}} . \\
& \cdot \int_{x}^{z_{\text {max }}^{\prime}} d z^{\prime} \frac{z^{\prime}\left(y z E-m_{\Lambda}\right)}{\left(t-\mu^{2}\right)^{2}} . \\
& \cdot F_{2}^{K}\left(\frac{x}{z^{\prime}}\right) g_{K N \Lambda}^{2}(t) .
\end{aligned}
$$

The range of the variables $\left(x, y, z, z^{\prime}\right)$ is to be discussed later in the section.

Note that, using the expression for $F_{2}(x)$, we may deduce the probability of finding a kaon within a proton; i.e. $F_{2}(x)$ can be expressed as the probability function convoluted with $F_{2}^{K}(x)$. Denote such probability function as $f_{K}^{p}\left(z^{\prime}\right)$. 


$$
\begin{aligned}
f_{K}^{p}\left(z^{\prime}\right) & \equiv \int G_{K}^{p}\left(z^{\prime}, z\right) d z \\
& =\int \frac{g_{K N \Lambda}^{2}}{4 \pi^{2}} m^{2} y E \frac{z^{\prime}\left(y z E-m_{\Lambda}\right)}{\left(t-\mu^{2}\right)^{2}} d z
\end{aligned}
$$

which is just (2) but expressed in different variables.

There are different ways to treat Sullivan processes. In addition to the above covariant method originally adopted by Sullivan [1], we proceed to use the alternative method developed by Drell, Levy, and Yan [20], which is essentially a perturbation theory in the infinite momentum frame, or better known as the light-cone perturbation theory. We shall see that the alternative method reproduces the same results (at least in the leading approximation). In light of the potential importance of (14) and (16), it is of interest to make certain that the same result can indeed be derived in such completely different formulation [20].

In the formulation developed by Drell, Levy, and Yan [20], one writes down the S-matrix, for a given interaction hamiltonian $H_{I}$,

$S=T e^{-i \int_{-\infty}^{\infty} d t H_{I}(t)}$,

with the matrix element given by

$$
\begin{aligned}
& <b|S| a>=-2 \pi i \delta\left(E_{b}-E_{a}\right)\left\{<b\left|H_{I}\right| a>\right. \\
& \quad+\sum_{n} \frac{<b\left|H_{I}\right| n><n\left|H_{I}\right| a>}{E_{a}-E_{n}+i \epsilon} \\
& \quad+\sum_{n_{1}, n_{2}} \frac{<b\left|H_{I}\right| n_{2}><n_{2}\left|H_{I}\right| n_{1}><n_{1}\left|H_{I}\right| a>}{\left(E_{a}-E_{n 1}+i \epsilon\right)\left(E_{a}-E_{n 2}+i \epsilon\right)} \\
& +\cdots\} .
\end{aligned}
$$

Some general characteristics of this formulation are [20]: Although each term is not covariant, the complete answer to a given order in perturbation series must be. The particle content of each term is clear, offering intuitive interpretations. At each vertex the three momenta are conserved, although the energy is not. A virtual particle is always on its mass shell, but the intermediate state may not have the same energy as the initial or final state.

The physical or dressed state $\mid A^{\prime}>$ of a particle can be expressed in terms of the bare state $\mid A>$ as follows [20],

$$
\left|A^{\prime}>=U\right| A>, \quad U=T e^{-i \int_{-\infty}^{0} d t H_{I}(t)} .
$$

Expanding $U$ to lowest order, we obtain

$$
\begin{aligned}
\mid A^{\prime}>= & \sqrt{Z}\left|A>+\sum_{B, C} \int d^{3} p_{B} d^{3} p_{C}\right| B, C> \\
& \cdot<B, C\left|(-i) \int_{-\infty}^{0} d t H_{I}(t)\right| A> \\
= & \sqrt{Z} \mid A>+ \\
& +\sum_{B, C} \int d^{3} p_{B} d^{3} p_{C} \frac{1}{E_{A}-E_{B}-E_{C}} \mid B, C>. \\
& \cdot<B, C\left|H_{I}\right| A>.
\end{aligned}
$$

It is convenient to express the matrix element of $H_{I}$ as

$$
\begin{aligned}
& <B, C\left|H_{I}\right| A>= \\
& \quad \frac{g V_{A \rightarrow B C}}{(2 \pi)^{\frac{3}{2}} \sqrt{2 E_{A} 2 E_{B} 2 E_{C}}} \delta^{3}\left(p_{A}-p_{B}-p_{C}\right) .
\end{aligned}
$$

If $B \neq C$, the probability of finding a particle in the final state $\mid B, C>$ is

$d P_{A \rightarrow B C}=\frac{\left|V_{A \rightarrow B C}\right|^{2}}{\left(E_{A}-E_{B}-E_{C}\right)^{2}} \frac{g^{2}}{(2 \pi)^{3}} \frac{d^{3} p_{B}}{2 E_{A} 2 E_{B} 2 E_{C}}$.

Note that a factor of $1 / 2$ must be included here, if $B, C$ are identical particles.

Simple expressions arise as we go over to the infinite momentum frame, i.e., by taking the following variables in the limit $P \rightarrow \infty$.

$p_{A}=\left(P+\frac{m_{A}^{2}}{2 P}, P, \mathbf{0}\right)$,

$p_{B}=\left(|z| P+\frac{p_{\perp}^{2}+m_{B}^{2}}{2|z| P}, z P, \mathbf{p}_{\perp}\right)$,

$p_{C}=\left(|1-z| P+\frac{p_{\perp}^{2}+m_{C}^{2}}{2|1-z| P},(1-z) P,-\mathbf{p}_{\perp}\right)$.

Here we see that at each vertex the three momentum is conserved while the energy is not, each particle is on mass shell, and $p_{B}, p_{C}$ carry the momenta of fractions $z$ and $1-z$ of the parent particle, respectively. The energy denominator in, e.g., (20) or (22) is

$$
\begin{array}{lr}
E_{A}-E_{B}-E_{C} & \\
=\frac{-p_{\perp}^{2}+z(1-z) m_{A}^{2}-(1-z) m_{B}^{2}-z m_{C}^{2}}{2 z(1-z) P}, & (0<z<1) \\
=-2(z-1) P, & (z>1) \\
=2 z P, & (z<0)
\end{array}
$$

so that the relevant region for $z$ is between 0 and 1 . Substituting these expressions back into (22), we find

$$
\begin{aligned}
& d P_{A \rightarrow B C}=\frac{g^{2}}{16 \pi^{2}} . \\
& \cdot \frac{z(1-z)\left|V_{A \rightarrow B C}\right|^{2}}{\left[p_{\perp}^{2}-m_{A}^{2} z(1-z)+m_{B}^{2}(1-z)+m_{C}^{2} z\right]^{2}} d z d p_{\perp}^{2} .
\end{aligned}
$$

Using the form for the $K N \Lambda$ vertex,

$V_{A \rightarrow B C}=i \bar{u}\left(p_{\Lambda}\right) \gamma_{5} u\left(p_{N}\right)$,

we obtain the spin average

$$
\begin{aligned}
\sum_{\text {spin av }}\left|V_{A \rightarrow B C}\right|^{2} & =-\frac{1}{2} \operatorname{Tr}\left(\hat{p}_{\Lambda}+m_{\Lambda}\right) \gamma_{5}\left(\hat{p}_{N}+m_{N}\right) \gamma_{5} \\
& =\frac{1}{1-z^{\prime}}\left\{p_{\perp}^{2}+\left[m_{\Lambda}-\left(1-z^{\prime}\right) m_{N}\right]^{2}\right\}
\end{aligned}
$$

with $z^{\prime}$ and $1-z^{\prime}$ the momentum fractions carried by $K$ and $\Lambda$, respectively. (Here we use $z^{\prime}$ in order not to confuse with $z=E_{\Lambda} / \nu$.) We thus obtain the probability of finding a kaon with longitudinal momentum fraction $z^{\prime}$ and transverse momentum $\mathbf{p}_{\perp}$.

$$
\begin{aligned}
& d P_{p \rightarrow K \Lambda} \equiv G_{K}^{p}\left(z^{\prime}, p_{\perp}^{2}\right) d z^{\prime} d p_{\perp}^{2} \\
& =\frac{g_{K N \Lambda}^{2}}{16 \pi^{2}} . \\
& \cdot \frac{z^{\prime}\left(p_{\perp}^{2}+\left(m_{\Lambda}-\left(1-z^{\prime}\right) m_{p}\right)^{2}\right)}{\left(p_{\perp}^{2}-m_{p}^{2} z^{\prime}\left(1-z^{\prime}\right)+m_{K}^{2}\left(1-z^{\prime}\right)+m_{\Lambda}^{2} z^{\prime}\right)^{2}} d z^{\prime} d p_{\perp}^{2} .
\end{aligned}
$$

In the light cone language, we use $P^{+}$to denote $E+P_{3}$. We note that the ratio of any two $P^{+}$is invariant under Lorentz boost in the $z$ direction. Thus, we have 


$$
1-z^{\prime}=\frac{P_{\Lambda}^{+}}{P_{p}^{+}}=\frac{1}{m_{p}}\left(E_{\Lambda}-\sqrt{E_{\Lambda}^{2}-m_{\Lambda}^{2}-p_{\perp}^{2}}\right) .
$$

The last equality is obtained by going over to the rest frame. In order to compare the result with (14) and (16), we change the variables $\left(z^{\prime}, p_{\perp}^{2}\right) \rightarrow\left(z^{\prime}, z\right)$.

$$
\begin{aligned}
p_{\perp}^{2} & =2 m_{p} \nu z\left(1-z^{\prime}\right)-m_{\Lambda}^{2}-m_{p}^{2}\left(1-z^{\prime}\right)^{2}, \\
\frac{\partial\left(z^{\prime}, p_{\perp}^{2}\right)}{\partial\left(z^{\prime}, z\right)} & =\left|\begin{array}{cc}
1 & 0 \\
\cdots & 2 m_{p} \nu\left(1-z^{\prime}\right)
\end{array}\right|=2 m_{p} \nu\left(1-z^{\prime}\right) . \\
G_{K}^{p}\left(z^{\prime}, z\right) & =G_{K}^{p}\left(z^{\prime}, p_{\perp}^{2}\right)\left|\frac{\partial\left(z^{\prime}, p_{\perp}^{2}\right)}{\partial\left(z^{\prime}, z\right)}\right| \\
& =\frac{g_{K N \Lambda}^{2}}{16 \pi^{2}} \frac{4 m_{p}^{2} \nu z^{\prime}\left(\nu z-m_{\Lambda}\right)}{\left(2 m_{p} \nu z-m_{p}^{2}+m_{K}^{2}-m_{\Lambda}^{2}\right)^{2}} .
\end{aligned}
$$

It is straightforward to verify that this last result is in fact identical to (17) which is obtained in Sullivan's original approach. The cross section may be obtained as follows,

$d \sigma=G_{K}^{p}\left(z^{\prime}, z\right) d z^{\prime} d z d \sigma(e K \rightarrow e X)$,

with $d \sigma(e K \rightarrow e X)$ the cross section that the electron hits the kaon with momentum fraction $z^{\prime}$ of the proton. We may use the formulae obtained earlier for $e N \rightarrow e X$ to describe $e K \rightarrow e X$ by using suitable definition of $x$ and $y$. Denoting them as $x^{\prime}$ and $y^{\prime}$, we have

$$
\begin{aligned}
x^{\prime} & \equiv \frac{Q^{2}}{2 p_{K} \cdot q}=\frac{Q^{2}}{2 z^{\prime} p \cdot q}=\frac{x}{z^{\prime}} \\
y^{\prime} & \equiv \frac{p_{K} \cdot q}{p_{K} \cdot p_{e}}=\frac{p \cdot q}{p \cdot p_{e}}=y
\end{aligned}
$$

Here $p_{K}, p_{e}, p$ represent the momenta of the kaon, the electron and the proton, respectively. We have

$$
\begin{aligned}
d \sigma(e K \rightarrow e X) & =\frac{2 \pi \alpha^{2}}{Q^{2} x^{\prime} y^{\prime}}\left[1+\left(1-y^{\prime}\right)^{2}\right] F_{2}^{K}\left(x^{\prime}\right) d x^{\prime} d y^{\prime} \\
& =\frac{\pi \alpha^{2}}{m E x^{2} y^{2}}\left[1+(1-y)^{2}\right] F_{2}^{K}\left(\frac{x}{z^{\prime}}\right) d x d y
\end{aligned}
$$

To sum up, we obtain

$$
\begin{aligned}
& \frac{d \sigma}{d x d y d z d z^{\prime}} \\
& \quad=G_{K}^{p}\left(z^{\prime}, z\right) \frac{\pi \alpha^{2}}{m E x^{2} y^{2}}\left[1+(1-y)^{2}\right] F_{2}^{K}\left(\frac{x}{z^{\prime}}\right),
\end{aligned}
$$

with $G_{K}^{p}\left(z^{\prime}, z\right)$ specified by (28). Note that the ranges for the variables $x, y, z$, and $z^{\prime}$ may be obtained from the constraints

$x \leq z^{\prime} \leq 1$,

$0 \leq p_{\perp}^{2} \leq E_{\Lambda}^{2}-m_{\Lambda}^{2}$.

Upon changing the variables from $\left(z^{\prime}, p_{\perp}^{2}\right)$ to $\left(z^{\prime}, z\right)$, the constraints become

$x \leq z^{\prime} \leq 1$,

$1 \geq z \geq \frac{1}{2 m_{p} E y}\left[\frac{m_{\Lambda}^{2}}{1-z^{\prime}}+m_{p}^{2}\left(1-z^{\prime}\right)\right] \equiv f\left(z^{\prime}\right)$.

The minimum of $f\left(z^{\prime}\right)$ occurs at $z^{\prime}=1-m_{\Lambda} / m_{p}<0$. To integrate the cross section of (30) in the order of $z^{\prime}, z, y$, and $x$, the corresponding integration region is specified by $x \leq z^{\prime} \leq 1-\frac{1}{m_{p}}\left(E y z-\sqrt{E^{2} y^{2} z^{2}-m_{\Lambda}^{2}}\right)$

$1 \geq z \geq \frac{1}{2 m_{p} E y}\left[\frac{m_{\Lambda}^{2}}{1-x}+m_{p}^{2}(1-x)\right]=f(x)$,

$1 \geq y \geq \frac{1}{2 m_{p} E}\left[\frac{m_{\Lambda}^{2}}{1-x}+m_{p}^{2}(1-x)\right]$,

$0 \leq x \leq 1-\frac{1}{m_{p}}\left(E-\sqrt{E^{2}-m_{\Lambda}^{2}}\right)$.

Note that (30) is the same as (16), except being expressed in terms of slightly different set of variables. In other words, the alternative formulation yields the same expression for the cross section as in the original Sullivan approach. In the present approach, we find that, by choosing the arguments to be $\left(z^{\prime}, p_{\perp}^{2}\right)$, the probability functions of the kaon and $\Lambda$ are the same. That is, $G_{K}^{p}\left(1-z^{\prime}, p_{\perp}^{2}\right)=G_{\Lambda}^{p}\left(z^{\prime}, p_{\perp}^{2}\right)$. Such aspect is by no means transparent in the original Sullivan approach.

To close this section, we turn to the standard fragmentation picture as illustrated by Fig. 1b. Denote by $D_{q}^{\Lambda}(z) d z$ the probability of quark $q$ fragmenting into $\Lambda$ of momentum fraction between $z$ and $z+d z$ [16]. The formula for $d \sigma / d x d y d z$ in the fragmentation picture is given by

$$
\begin{aligned}
\frac{d^{3} \sigma}{d x d y d z}= & \frac{\pi \alpha^{2}}{m E x^{2} y^{2}}\left[1+(1-y)^{2}-\right. \\
& \left.-\frac{m}{E} x y\right] \sum_{q} e_{q}^{2} x f_{q}(x) D_{q}^{\Lambda}(z) \\
\approx & \frac{2 \pi \alpha^{2}}{Q^{2} x y}\left[1+(1-y)^{2}\right] \sum_{q} e_{q}^{2} x f_{q}(x) D_{q}^{\Lambda}(z) .
\end{aligned}
$$

\section{Sample Numerical Results}

To describe the numerical results, we write the cross section of (32) as $d \sigma^{(2)}$ for the fragmentation picture (Fig. 1b) in contradistinction with $d \sigma^{(1)}$, (30) as from the Sullivan process (Fig. 1a). The ratio $d \sigma^{(1)} / d \sigma^{(2)}$ is then given by

$$
\frac{\frac{d \sigma^{(1)}}{d x d y d z}}{\frac{d \sigma^{(2)}}{d x d y d z}}=\frac{\int_{x}^{z_{\text {max }}^{\prime}} d z^{\prime} F_{2}^{K}\left(\frac{x}{z^{\prime}}\right) G_{K}^{p}\left(z^{\prime}, z\right)}{\sum_{q} e_{q}^{2} x f_{q}^{p}(x) D_{q}^{\Lambda}(z)} .
$$

We choose to present the numerical results on this ratio as a gauge of the relative importance between these two processes, mainly because the current crude status regarding the fragmentation functions $[6,16,21,22]$ does not give us enough confidence in the calculated absolute magnitudes of these cross sections.

To obtain sample numerical results, it is sufficient to use the standard simple ansatz $[16,6,21,22]$ :

$$
\begin{aligned}
& \left\{\begin{array}{l}
x f_{u}^{K^{+}}(x)=x f_{\bar{s}}^{K^{+}}(x)=v^{K^{+}}(x)+s^{K^{+}}(x) \\
x f_{\bar{u}}^{K^{+}}(x)=x f_{d}^{K^{+}}(x)=x f_{s}^{K^{+}}(x)=x f_{\bar{d}}^{K^{+}}(x)=s^{K^{+}}(x)
\end{array}\right\} \\
& \left\{\begin{array}{l}
x f_{u}^{p}(x)=u^{p}(x)+s^{p}(x), x f_{d}^{p}(x)=d^{p}(x)+s^{p}(x) \\
x f_{\bar{u}}^{p}(x)=x f_{\bar{d}}^{p}(x)=2 x f_{s}^{p}(x)=2 x f_{\bar{s}}^{p}(x)=s^{p}(x)
\end{array}\right\}
\end{aligned}
$$




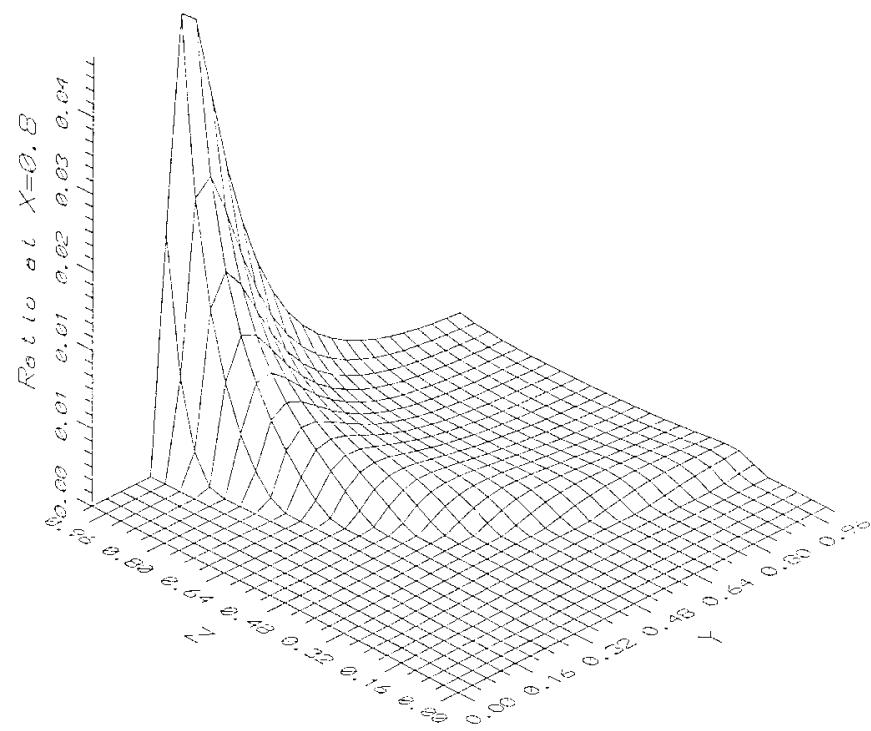

Fig. 2. The ratio given by (33) is shown as a function of $y$ and $z$ for a fixed value of $x(x=0.8)$. The ridge is specified by the curve $y z=\frac{1}{2 m_{p} E}\left[\frac{m_{\Lambda}^{2}}{1-x}+\right.$ $\left.m_{p}^{2}(1-x)\right]$. On one side of this curve, the ratio drops to zero because it is out of the range mentioned above (31). Note that the maximum occurs at $z=1$

$$
\begin{aligned}
u^{p}(x) & =A_{u}^{p} x^{\alpha_{u}^{p}}(1-x)^{\beta_{u}^{p}}, \quad d^{p}(x)=A_{d}^{p} x^{\alpha_{d}^{p}}(1-x)^{\beta_{d}^{p}}, \\
s^{p}(x) & =A_{s}^{p}(1-x)^{\gamma^{p}} \\
v^{K^{+}}(x) & =A^{K^{+}} x^{\alpha^{K^{+}}}(1-x)^{\beta^{K^{+}}} \\
s^{K^{+}}(x) & =A_{s}^{K^{+}}(1-x)^{\gamma^{K^{+}}} .
\end{aligned}
$$

The various parameters are fitted from experiments and found to be [21]

$$
\begin{aligned}
& A^{K^{+}}=0.4942, \quad A_{s}^{K^{+}}=0.027 ; \\
& \alpha^{K^{+}}=0.33, \quad \beta^{K^{+}}=1.17, \quad \gamma^{K^{+}}=9.29 ; \\
& \alpha_{u}^{p}=\alpha_{d}^{p}=0.57, \quad \beta_{u}^{p}=\beta_{d}^{p}-1=3.28, \quad \gamma^{p}=9.33 ; \\
& A_{u}^{p}=2.906, \quad A_{d}^{p}=1.651, \quad A_{s}^{p}=0.04617 .
\end{aligned}
$$

As for the fragmentation functions, we use the simple form (adapted from [22]):

$z D_{u}^{\Lambda}=z D_{d}^{\Lambda}=z D_{s}^{\Lambda}=B_{s}(1-z)^{4}+B_{v} z(c-z)^{3}$,

$z D_{\bar{u}}^{\Lambda}=z D_{\bar{d}}^{\Lambda}=z D_{\bar{s}}^{\Lambda}=B_{s}(1-z)^{4}$.

$$
B_{s}=0.1, \quad B_{v}=0.2, \quad c=\frac{11}{9} \text {. }
$$

We take the dipole form for the $K N \Lambda$ coupling [12].

$g_{K N \Lambda}(t)=g_{K N \Lambda}^{0}\left(\frac{\Lambda^{2}-m_{K}^{2}}{\Lambda^{2}-t}\right)^{2}$,

with the cutoff $\Lambda$ taken to be $1400 \mathrm{MeV}$.

The sample numerical results are shown in Figs. 2-5. For the sake of illustration, we take the incident electron energy $E$ to be $20 \mathrm{GeV}$.

In Fig. 2, the ratio is shown as a function of $y$ and $z$ for a fixed value of $x(x=0.8)$. It is seen that there is a ridge along the curve

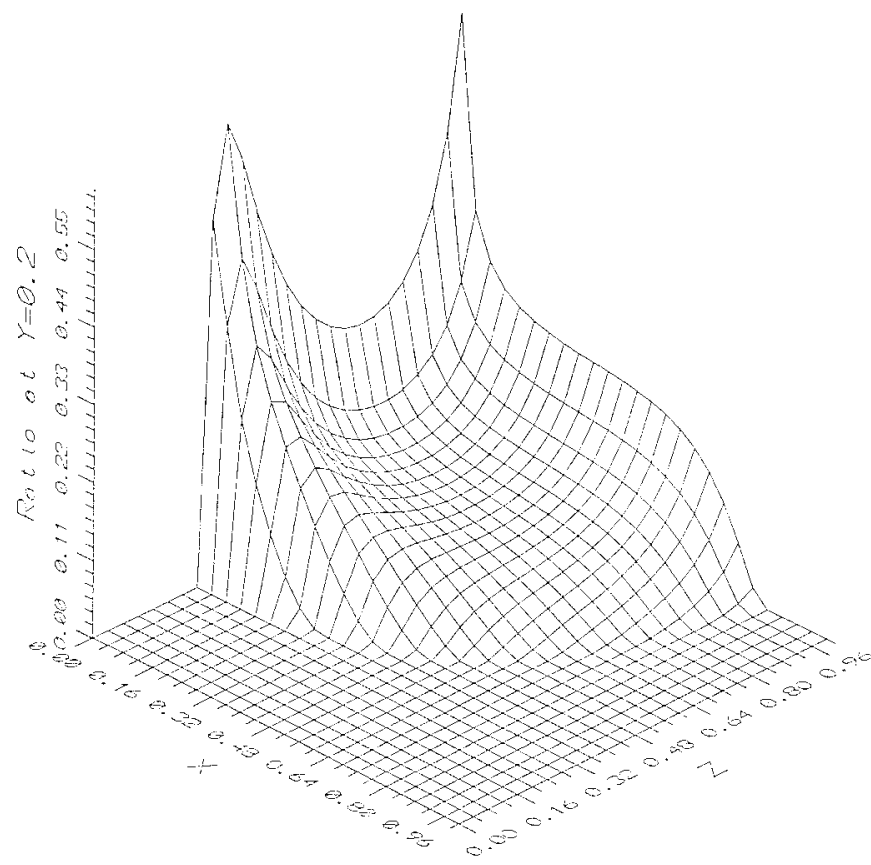

Fig. 3. The ratio given by (33) is shown as a function of $x$ and $z$ for a fixed value of $y(y=0.2)$

$y z=\frac{1}{2 m_{p} E}\left[\frac{m_{\Lambda}^{2}}{1-x}+m_{p}^{2}(1-x)\right]$

On one side of this curve, the ratio drops to zero because it is out of the range mentioned above (31). Note that the maximum occurs at $z=1$.

In Fig. 3, the ratio is shown as a function of $x$ and $z$ for a fixed value of $y(y=0.2)$. Here the parameters are chosen such that the ratio is clearly of numerical importance in a visible part of the $(x, z)$ parameter space.

In Fig. 4, the ratio is shown as a function of $x$ and $y$ for a fixed value of $z(z=0.1)$. It is seen that, when $z$ is fixed, the maximum occurs at $x=0$. The global maximum may be located by plotting the curve with $x=0$ and $z=1$ and is found to be located at $y=0.075$. In general, we find that the global maximum occur at $x=0, z=1$ and $y E=1.5 \mathrm{GeV}$ when we change the electron energy $E$. The maximum value for the ratio is approximately 24 (which is rather large) and is independent of $E$. However, such point is mostly academic (and likely of little experimental interest) since the peak is fairly sharp and narrow. As the incident electron energy $E$ increases, the peak becomes sharper; that is, the ratio at the points other than the peak decreases rapidly. This is because the coupling goes to zero as $E$ becomes infinity except for the region with small $y z E$.

In Fig. 5a, the double differential cross section $\frac{d \sigma}{d x d y}$ for the Sullivan process, as obtained from integrating (30) subject to (31), is plotted as a function of $x$ and $y$. The unit is $10^{-15} \mathrm{MeV}^{-2}$. For a given value of $x$, the cross section vanishes at small $y$ (where it is kinematically forbidden), rises rapidly once $y$ crosses the threshold, reaches at some peak value and then falls off as $y \rightarrow 1$. In Fig. 5b, we display the same differential cross section at a specific value of $x(=0.01)$ as a function of $y$. The dots indicate the points which we have 


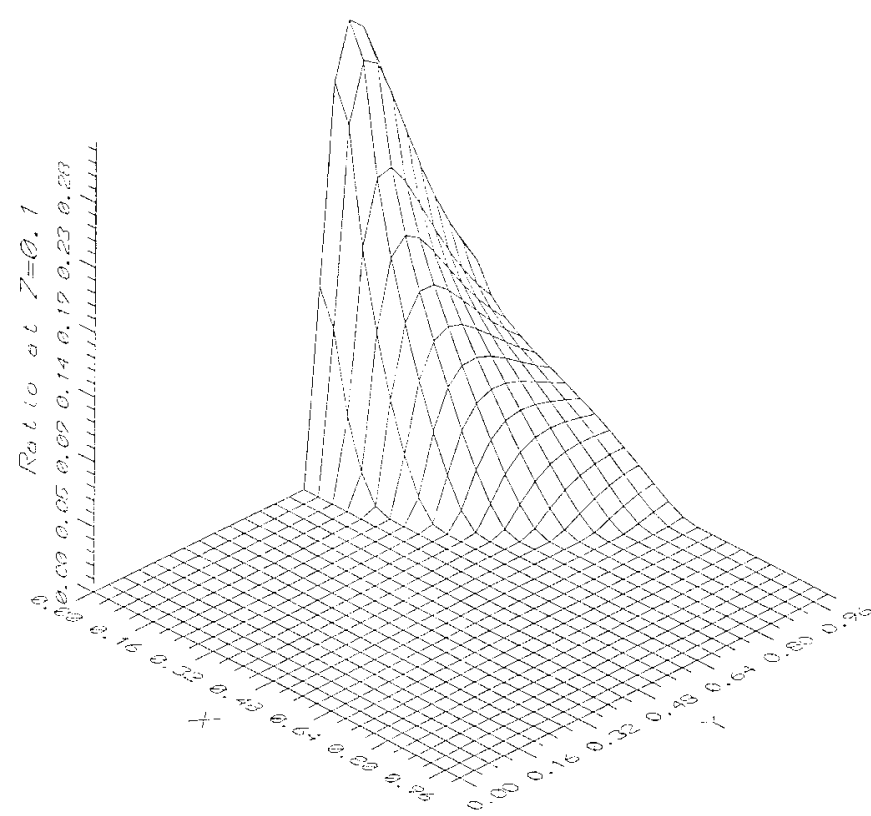

Fig. 4. The ratio given by (33) is shown as a function of $x$ and $y$ for a fixed value of $z(z=0.1)$. Note that, when $z$ is fixed, the maxinum occurs at $x=0$
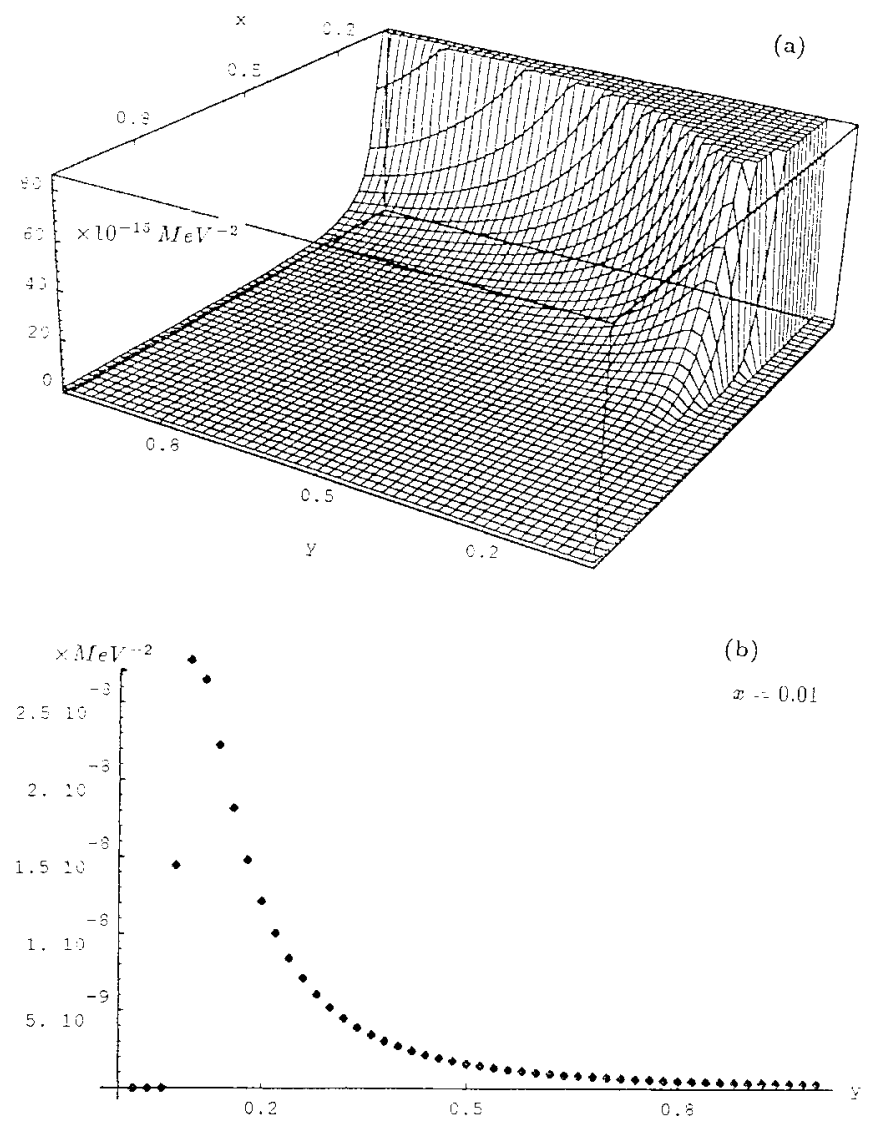

Fig. 5.a,b. The double differential cross section $\frac{d \sigma}{d x d y}$ for the Sullivan process, as obtained from integrating (30) subject to (31), is shown: a as a function of $x$ and $y$ (in units of $10^{-15} \mathrm{MeV}^{-2}$ ) and $\mathbf{b}$ shown as a function of $y$ for $x=0,01$ performed the integrations (over $z^{\prime}$ and $z$ ) to obtain the predicted cross sections.

Unlike Figs. 2-4 for which the ratio of the cross sections is shown, Figs. 5a and 5b gives the cross section itself. Yet, it should be regarded as semi-quantitative owing to the uncertainties in connection with (34)-(37). Nevertheless, it may serve as the basis for analyzing the feasibility of the proposed experiment. Assuming a luminosity of $10^{32} \mathrm{~cm}^{-2} \mathrm{~s}^{-1}$ (and with $100 \%$ detection efficiency), it takes a day of running time to see about three events for a cross section of $10^{-15} \mathrm{MeV}^{-2}$. The estimates shown in Figs. 5a and 5b are clearly quite encouraging in terms of the experimental feasibility.

The sample numerical results shown by Figs. 2-5 are very suggestive. To confirm the potential importance of the generalized Sullivan process, Fig. 1a, in the semi-inclusive $\Lambda$ production or, equivalently, to identify the kinematic region where the standard fragmentation picture, Fig. 1b, may not be adequate, it is essential to identify those events which have relatively small $y$ (say, $0.05 \leq y \leq 0.2$ with $y \equiv \nu / E$ the fraction of the energy transfer), large $z$ (say, $z \geq 0.6$ - most of the energy transferred to the proton is again carried away by $\Lambda$ ), and small $x$ (say $0.05 \leq x \leq 0.4$ ). It is expected from Figs. 2-5 that events in this region arise partially from the mechanism associated with the generalized Sullivan process, Fig. 1a. An appropriate analysis of the data in this region must take into account both the mechainsms - the contribution from the generalized Sullivan process Fig. 1a and that from the standard fragmentation picture Fig. 1b. Failing to include the contribution from the generalized Sullivan process, one would obtain fragmentation functions which would in general over-predict cross sections elsewhere.

The proposed experiment to confirm the major conclusion of the present paper is not an easy one but should be feasible. Unlike the case of non-strange hadrons [18], semiinclusive production of $\Lambda$ in general has smaller cross sections in the fragmentation picture but has much larger signal-tobackground ratios as far as the generalized Sullivan process is concerned. Detection of $\Lambda$ through its $N \pi$ decay modes also offers an unambiguous experimental signal. In fact, polarization of $\Lambda$ can also be measured without much difficulty, although predictions on the $\Lambda$ spin is left as a subject for future investigation. In our opinion, more efforts should be dedicated to measurements of semi-inclusive hadron production cross sections so that a clear picture towards the various fragmentation functions, a concept closely related to parton distributions, may be obtained.

\section{Summary}

In this paper, we have investigated the proposal of using semiinclusive $\Lambda$ production with a high energy electron or muon beam as a means of testing the suggestion that the generalized Sullivan process, in which the virtual photon may strike and smash the meson in the cloud surrounding the target proton, may play an important role in deep inelastic scattering (DIS) by charged leptons. We have considered two different ways to formulate the Sullivan process as illustrated in Fig. 1a and obtain the same result. Our sample numerical results, Figs. $2-5$,indicate that, in the kinematic region characterized typi- 
cally by small $x$, large $z$, and a small specific range of $y$, the contribution from Sullivan processes Fig. 1a is of numerical importance as compared to that due to the fragmentation process Fig. 1b. It is clear that semi-inclusive $\Lambda$ production with a high energy electron or muon beam may offer an alternative means of testing the importance of the generalized Sullivan process.

This work was supported in part by the National Science Council of the Republic of China (NSC86-2112-M002-010Y).

\section{References}

1. J. D. Sullivan, Phys. Rev. D5, 1732 (1972)

2. A. W. Thomas, Phys. Lett. 126B, 97 (1983); L. L. Frankfurt, L. Mankiewicz, and M.I. Strikman, Z. Phys. A - Atomic Nuclei 334, 343 (1989); S. Kumano, Phys. Rev. D43, 59 (1991)

3. J. J. Aubert et al., Phys. Lett. 163B, 275 (1983)

4. A. Bodek et al., Phys. Rev. Lett. 50, 1431 (1983); 51, 534 (1983); R.G. Arnold et al., Phys. Rev. Lett. 52, 727 (1984)

5. G. Bari et al., Phys. Lett. 163B, 282 (1985)

6. L. Montanet et al., Particle Data Group, Phys. Rev. D50, 1173 (1994)

7. C. H. Llewellyn Smith, Phys. Lett. B128, 107 (1983)
8. M. Ericson and A. W. Thomas, Phys. Lett. B128, 112 (1983); M. Ericson, Prog. Part. Nucl. Phys. 11, 277 (1984); A. W. Thomas, Prog. Part. Nucl. Phys. 11, 325 (1984)

9. D. Stump, G. F. Bertsch, and J. Pumplin, in "Hadron Substructure in Nuclear Physics" (AIP Conf. Proc. No. 110), Eds. W-Y. P. Hwang and M. H. Macfarlane (AIP, New York, 1984), p. 339

10. E.L. Berger, F. Coester, and R. B. Wiringa, Phys. Rev. D29, 398 (1984)

11. E. M. Henley and G. A. Miller, Phys. Lett. B251, 453 (1990); S. Kumano, Phys. Rev. D43, 3067 (1991); S. Kumano and J. T. Londergan, Phys. Rev. D44, 717 (1991); A. Signal, A. W. Schreiber, and A. W. Thomas, Mod. Phys. Lett. A6, 271 (1991); J. Stern and G. Clement, Phys. Lett. B264, $426(1991)$

12. W-Y. P. Hwang, J. Speth, and G. E. Brown, Z. Phys. A339, 383 (1991)

13. W-Y. P. Hwang and J. Speth, Phys. Rev. D46, 1198 (1992)

14. K. Gottfried, Phys. Rev. Lett. 18, 1154 (1967)

15. P. Amaudruz et al., Phys. Rev. Lett. 66, 2712 (1991)

16. J. Ashman et al., Nucl. Phys. B328, 1 (1989); B. Adeva et al., Phys. Lett. B302, 533 (1993); P. L. Anthony et al., Phys. Rev. Lett. 71, 959 (1993); K. Abe et al., Phys. Rev. Lett. 75, 25 (1995)

17. C.-H. Chung and W-Y. P. Hwang, Phys. Rev. D49, 2221 (1994); J. Speth, private communication

18. C. I. Korpa, A.E.L. Dieperink, and O. Scholten, Z. Phys. A343, 461 (1992); G. D. Bosveld, A. E. L. Dieperink, and O. Scholten, Phys. Rev. C45, 2616 (1992)

19. R. D. Field and R.P. Feynmen, Phys. Rev. D15, 2590 (1977); Nucl. Phys. B136, 1 (1978)

20. S. D. Drell, D. J. Levy and T.-M. Yan, Phys. Rev. D1, 1035 (1970)

21. J.Badier et al., Z. Phys. C18, 281 (1983)

22. R.Badier et al., Z. Phys. C2, 265 (1979) 